among vitamin $\mathrm{B}_{2}$-deficient rats, but not on an extensive scale.

The above would indicate in outline the complexity of what is called 'vitamin $\mathrm{B}_{2}$ '. At least four factors appear to be involved-the flavine, the heatstable factor, the anti-dermatitis factor and the anticataract factor, though it is not improbable that two or more of them may be identical. We would suggest that provisionally the term 'vitamin $\mathrm{B}_{2}$ ' be reserved for the entire complex, which supplements the usual vitamin $\mathrm{B}_{2}$-deficient diet for the promotion of good growth in rats. The other factors may be indicated by their special characteristics or methods of assayfor example, flavine, anti-dermatitis factor, anticataract factor, ete.

We shall, perhaps, know then where we are. B. C. GuHa.

Biochemical Laboratory,

Bengal Chemical and Pharmaceutical Works Ltd., Calcutta.

Feb. 4.

1 Guha, Brit. Med. J., 2, 53 ; 1931.

'Guha and Biswas, Current Science, 3, 300; 1935. Ber. deutsch. chem. Gesell., in press.

${ }^{3}$ Chick and Copping, Chemistry and Industry, 53, 874; 1934.

Booher, Blodgett and Page, J. Biol. Chem., 107, 599; 1934.

Elvehjem and Koehn, NATURE, 134, Dec. 29, 1934

'Langston and Day, Southern Med. J.. 26, 128; 1933.

\section{Reproduction and Cancer}

Prof. E. C. Dodos and Dr. J. W. $\operatorname{Cook}^{1}$ have published much information regarding the chemical and pharmacological relationship between the sex hormones such as cestrin and certain carcinogenic hydrocarbons. They have established in those respects an interesting connexion between the growth changes of the uterus and those observed in certain types of cancer.

There are other interesting facts concerning the two processes. Thus carbon monoxide gas renders mice sterile ${ }^{2}$, when breathed in concentrations $(0 \cdot 25$ per cent) which do not interfere with the general growth of the body of mice acclimatised gradually; in the same concentration the gas retards both rate of growth of mouse carcinoma No. 63 (Bashford's tumour) and development of tar cancer ${ }^{3}$.

Again, embryonic skin of mouse is--equally with placental tissue - the most potent agent in rendering mice immune to transplantable and spontaneous tumours ${ }^{4}$; it is possible that this epithelium manufactures 'immune bodies' as the result of œestrogenic and uterine activity. The interesting question is why these 'immune bodies' should be most concentrated in the embryonic skin as compared with other embryonic tissues; it may be due to the origin of the skin from ectoderm which in the early stages lies nearest to the uterine decidua, a tissue influenced by œstrin.

If œestrin is responsible, directly or indirectly, for production of natural immunity, then we should expect cancer to be most prevalent when the production of cestrin ceases. This is the case, at any rate in the female, since cancer is most prevalent after the menopause.

National Institute for Medical Research,

\section{J. Argyll Campbell.}

N.W.3.

Feb. 12 .

1 Ann. Rep. Brit. Emp. Cancer Campaign, 11, 12 ; 1934

${ }^{2}$ Quart. J. Exp. Physiol., 24, 271; 1934.

- Murphy, J. B., Bull. Johns Hopkins Hosp., 56, $1 ; 1935$.

\section{Raman Spectrum of Gaseous Carbon Disulphide}

The $\Delta \nu=655 \mathrm{~cm}^{-1}$ Raman band of carbon disulphide was photographed, the substance being in the state of vapour. Fig. 1 shows a spectrogram taken with a glass $F / 6$ two-prism thermostated spectrograph, using a $60 \mathrm{~cm}$. vapour column at about 4.5 atm. pressure, irradiated by a mercury arc, the radiation from which was filtered through a dilute solution of potassium chromate to cut off the ultra-violet, avoiding photochemical decomposition and visible fluorescence of the vapour.

The exposure of the spectrogram reproduced was 100 hours on Agfa Isochrom plate, during which the 'Telex' glass Raman tube, air-freed and containing some purified carbon disulphide liquid in its tail annex, was maintained thermostatically at $100^{\circ} \mathrm{C}$., the irradiated portion and the sealed-in plane-parallel window at slightly higher temperatures.

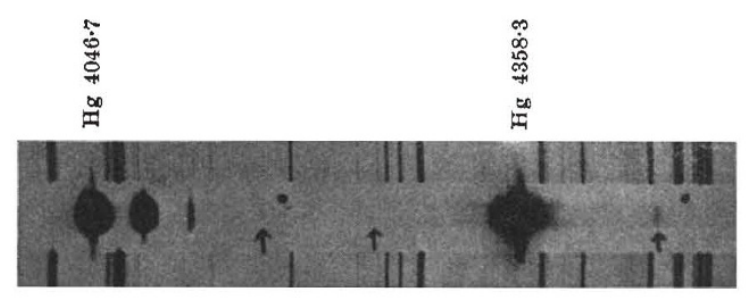

Fic. 1. Raman spectrum of carbon disulphide vapour. Copper arc above and below.

The satellites found in the spectrum of the liquid ${ }^{1}$ and interpreted as due to transitions from excited vibrational states are not resolved, even if present, on our plates, with a linear dispersion $20 \mathrm{~A}$. per $\mathrm{mm}$. at $\lambda 4358$ and a large slit width, $0.1 \mathrm{~mm}$. According to the most recent and accurate data for the liquid ${ }^{2}$ the centre of gravity of the two stronger component bands is shifted by $655 \cdot 0 \mathrm{~cm} .^{-1}$ from the exciting frequency.

The shift of the intensity maximum in the gaseous spectrum was observed as two Stokes lines of mercury $\lambda \lambda 4047$ and 4358 , and an anti-Stokes line of the latter as marked by an arrow in Fig. 1. The measured value from the two Stokes lines on the best plate (reproduced) is $655 \pm 1 \mathrm{~cm}^{-1}$, and is the same as the liquid shift. The negligible influence of the change of state upon the molecular vibration frequency of carbon disulphide may be expected from the smallness of dipole moment of the substance.

The intensity ratio of the $\Delta v=796 \mathrm{~cm}^{-1}$ band to that of $\Delta v=655 \mathrm{~cm} .^{-1}$ appears to be much lower in the spectrum of the gas than in that of the liquid. Excepting a very faint and doubtful blackening, no measurable trace of the former band has been recorded above background level up to 125 hours exposure (dotted parts in Fig. 1) ; whereas with the liquid, as high as $1: 3$ is reported for the above intensity ratio under ordinary conditions of observa. tion $^{2}$. It is intended to measure photometrically the value of it on a denser and sharper plate in the near future.

Institute of Physical and

Sunao Imanishi.

Chemical Research,

Komagome, Hongo, Tokyo. Nov. 30 .

${ }^{1} \mathrm{P}$. Krishnamurti and others. See literature in (2).

A. Langseth, J. U. Sørensen and J. R. Nielsen, J. Chem. Phys., 2, $402 ; 1934$. 